\title{
Controlled Bending of Microscale Au-Polyelectrolyte Brush Bilayers
}

\author{
Tim S. Kelby ${ }^{\dagger}$ and Wilhelm T. S. Huck $*,+$, \\ ${ }^{\dagger}$ Melville Laboratory for Polymer Synthesis, Department of Chemistry, University of Cambridge, \\ Lensfield Road, Cambridge CB2 1EW, U.K., and ${ }^{*}$ Radboud University Nijmegen, Institute for \\ Molecules and Materials, Heyendaalseweg 135, 6525 AJ Nijmegen, The Netherlands
}

Received March 22, 2010; Revised Manuscript Received May 4, 2010

\begin{abstract}
Free-standing Au-polyelectrolyte brush bilayer objects were fabricated by a facile route based on microcontact printing and chemical etching. Patterned poly(methacryloxyethyltrimethylammonium chloride) (PMETAC) brushes were grown on a gold-coated silicon wafer, which was etched to produce free-standing bilayer objects. These bilayers, produced with different thicknesses of Au, were imaged by optical microscopy in suspension in water and in $\mathrm{NaCl}$ solutions of varying concentrations. The radius of curvature of these objects was used to extract values for the surface stress induced by the brush in salted and salt-free regimes and to investigate the time scale of the brush swelling transition, demonstrating that this technique can be used to probe the mechanical properties of stimulus-responsive brushes.
\end{abstract}

\section{Introduction}

Polyelectrolyte brushes provide a facile route to robust stimulus-responsive surfaces: they are easily synthesized with good control over grafting density, thickness, and other parameters; ${ }^{1,2}$ they are easily patterned by microcontact printing;, ${ }^{3,4}$ and they have been shown to respond to a wide range of stimuli. ${ }^{2,5-9}$

The behavior of end-grafted polymer chains is governed by the balance between the chains' conformational entropy, which favors a random coil conformation, and interchain interactions, which may be entropic, electrostatic, or steric. ${ }^{10,11}$ Because of the constraint imposed on the chain ends by the grafting, these repulsive interchain interactions give rise to a mechanical stress within the brush. ${ }^{12-15}$

These mechanical stresses can be harnessed to create nanoscale actuators which deform in response to an environmental change. Polyelectrolyte brushes have been used, ${ }^{16-18}$ for example, to reversibly bend an AFM cantilever in response to electrochemical processes ${ }^{19}$ or to changing $\mathrm{pH}$ and salt concentration, ${ }^{20}$ neutral brushes responsive to solvent quality ${ }^{21}$ and temperature ${ }^{22}$ have been used in similar experiments. However, these systems are, by their nanoscale nature, difficult to observe and characterize, since the deformations produced are invisible to optical microscopy and often have a low signal-to-noise ratio due to the effects of thermal drift and fluctuations in solution conditions. ${ }^{23}$ The cantilever substrates also introduce an element of complication, since they consist of multiple layers of different materials, the properties of which must be known and modeled accurately.

In some previous studies, the strain within a polymer film has been determined from measurements of the curvature of microscale polymer-metal bilayer objects, ${ }^{24-26}$ demonstrating that it is possible to use bilayer curvature to calculate the strain within the stressed layer. This provides a convenient methodology for quantifying, understanding, and hence learning to control and harness the mechanical stresses in polymer brushes. By fabricating free-standing $\mathrm{Au}$-polyelectrolyte brush bilayer objects and measuring their curvature, the origins of these mechanical stresses can be better understood.

*Corresponding author. E-mail: wtsh2@cam.ac.uk,w.huck@science.ru.nl.

\section{Experimental Section}

Materials and Reagents. All chemicals were analytical reagent grade and obtained from Sigma-Aldrich and used as purchased unless otherwise specified. Copper(I) bromide was obtained with $\geq 99.995 \%$ purity (trace metals basis) from Sigma-Aldrich and stored under vacuum before use. Solvents were obtained from Fisher Scientific.

Methacryloxyethyltrimethylammonium chloride (METAC) was obtained as a $75 \%$ aqueous solution containing $600 \mathrm{ppm}$ monomethyl ether hydroquinone as inhibitor; the monomer was purified to remove the inhibitor by passing the solution through a basic alumina column (Brockmann grade I).

11-Mercaptoundecyl bromoisobutyrate (ATRP initiator thiol) was synthesized from 2-bromoisobutyryl bromide and mercaptoundecanol following a previously published procedure. ${ }^{27}$ DI water (resistivity $18.2 \mathrm{M} \Omega \cdot \mathrm{cm}$ ) was obtained using a Millipore Synergy UV purification system.

Characterization. AFM topography images were acquired with a Digital Instruments Dimension 3100 AFM (Veeco Instruments, Inc.) in tapping mode, using OMCL-AC series silicon probes (resonant frequency $\approx 300 \mathrm{kHz}, k \approx 42 \mathrm{~N} \mathrm{~m}^{-1}$, Olympus Corp.). The images were acquired and processed using the NanoScope software (Veeco Instruments, Inc.). Optical micrographs were acquired using a Nikon Eclipse ME600 optical microscope fitted with a Nikon DN100 digital net camera. Ellipsometric measurements were carried out with a fixed-angle spectroscopic ellipsometer ( $\alpha$-SE from J.A. Woollam Co., Inc.) using a homemade liquid cell. At least three measurements were taken at different points on each sample.

Preparation of Au-Modified Si Wafers. Silicon wafers were obtained from Compart Technology Ltd. (100 mm diameter, boron-doped, $\langle 100\rangle$ orientation), plasma oxidized for $10 \mathrm{~min}$ at $100 \mathrm{~W}$ in an Emitech Plasma Asher, and coated by deposition of chromium followed by gold in a BOC-Edwards Auto 500 thermal evaporation and RF sputter-coater, at a deposition rate of $\leq 0.1 \mathrm{~nm} \mathrm{~s}^{-1}$.

Preparation of Au-Polyelectrolyte Bilayer Objects. Au-coated silicon wafers were modified with patterned monolayers of initiator thiol by microcontact printing following previously reported procedures. ${ }^{27,28}$

For the growth of PMETAC, METAC (13.85 g, $67 \mathrm{mmol})$ was dissolved in a mixture of water $\left(4 \mathrm{~cm}^{3}\right)$ and isopropanol 


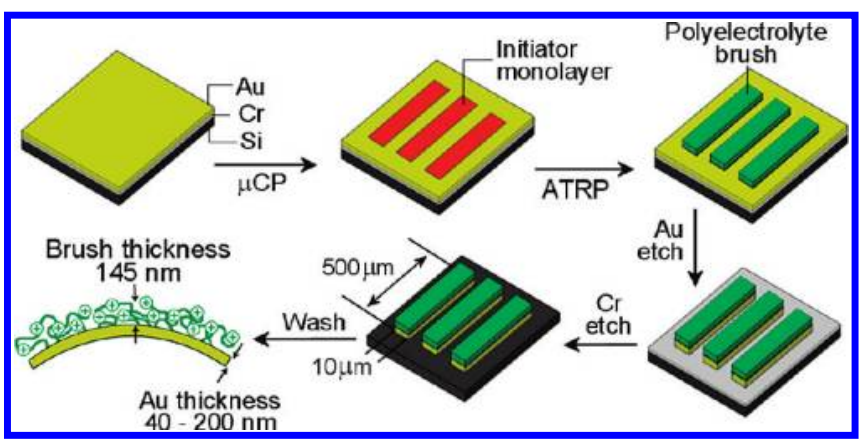

Figure 1. Fabrication of free-standing $\mathrm{Au}$-polymer bilayer objects.

(IPA) $\left(16 \mathrm{~cm}^{3}\right) .2,2^{\prime}$-Bipyridine (382 mg, $\left.2.4 \mathrm{mmol}\right)$ and copper(II) chloride $(6.7 \mathrm{mg}, 0.05 \mathrm{mmol})$ were added, and the mixture stirred and degassed for $1 \mathrm{~h}$, after which copper(I) bromide $(0.144 \mathrm{~g}, 1.0 \mathrm{mmol})$ was added to the solution.

Initiator-modified Au substrates were sealed in Radley tubes and degassed (three high-vacuum/ $\mathrm{N}_{2}$ cycles), and enough polymerization solution was added to each substrate to submerge it completely. The polymer-modified samples were removed after $375 \mathrm{~min}$ at room temperature, washed sequentially with DI water and acetone, and dried in flowing $\mathrm{N}_{2}$.

Etching of the substrate was carried out as follows, according to previously published procedures. ${ }^{29,30}$ Etching of the gold layer on the wafer was carried out by placing a drop of gold etchant (ratio by mass, 4:1:40 KI: $\mathrm{I}_{2}: \mathrm{H}_{2} \mathrm{O}$ ) on the patterned brush sample for $20 \mathrm{~s}$. The drop was then washed off the surface with DI water. Etching of the chromium layer was carried out by covering the sample with a droplet of chromium etchantcerium ammonium nitrate $(20 \mathrm{~g})$ in aqueous acetic acid solution $(100 \mathrm{~mL}, 0.6 \mathrm{M})$ - for $60 \mathrm{~min}$. This length of time was chosen to produce complete etching of the thickest $\mathrm{Cr}$ layers used. The $\mathrm{Au}$-polymer bilayer objects formed in this way were washed off the Si substrate with DI water; for experiments involving salt solutions, the objects were washed off with DI water, and then $1.0 \mathrm{M} \mathrm{NaCl}$ solution was added to produce the correct salt concentration.

Control samples were produced by synthesis of patterned brushes followed by $\mathrm{Au}$ etching as described above. The brushes were then removed by immersion in piranha solution ( $3: 1 \mathrm{v} / \mathrm{v}$ concentrated $\mathrm{H}_{2} \mathrm{SO}_{4} / 30 \%$ aqueous $\mathrm{H}_{2} \mathrm{O}_{2}$ solution) until bubbles of oxygen were no longer evolved, and the samples were immersed for $2 \mathrm{~h}$ in a $2 \mathrm{mM}$ ethanolic solution of the ATRP initiator thiol. Cr etching and wash-off were carried out as described above, producing strips of Au without a polymer layer, coated on one side with a SAM of ATRP initiator thiol.

\section{Results and Discussion}

Fabrication of Bilayer Objects. Free-standing linear $\mathrm{Au}$-polyelectrolyte bilayer objects were fabricated by a simple and readily repeatable method based on microcontact printing, followed by ATRP and then etching of the metal substrate (see Figure 1). Si wafers were modified, first with a thin layer of $\mathrm{Cr}$ and then with a thicker layer of $\mathrm{Au}$, by thermal evaporation; the Au layer thickness was systematically varied, and the $\mathrm{Cr}$ thickness was always kept at $10 \%$ of the Au thickness. The gold surface was microcontact printed with a monolayer of thiol ATRP initiator, patterned in $(10 \times 500) \mu \mathrm{m}$ rectangles with a $10 \mathrm{~mm}$ spacing. Patterned brushes of PMETAC were then grown from the initiator layer by ATRP in IPA/water, a method known to produce fast, controlled polymerization of hydrophilic monomers. ${ }^{31,32}$ Brush thickness in the dry state was measured by AFM, and samples were selected with a brush thickness of $145 \pm 5 \mathrm{~nm}$.
Following brush growth, the Au layer was etched to remove the gold film from between the polyelectrolyte, and then the Cr layer was etched completely away, leaving the Au-brush objects to be washed off the Si substrate. Etching of the Au layer for too long resulted in penetration of the brush by the etchant, resulting in some etching of the gold under the brush, which produced damaged bilayer objects which bent anisotropically.

Theoretical Treatment. In many previous studies on microcantilevers, the mechanical stresses that give rise to bending have been quantified using the Stoney equation, ${ }^{15,19,20,22}$ which relates the curvature of a cantilever to surface stress. However, Stoney's theory assumes a bilayer consisting of two homogeneous elastic solids, whereas the brush layer in our system produces no restoring force on deformation and hence cannot be modeled as elastic. Instead, following the approach of Sushko, ${ }^{15}$ we decouple the mechanical deformation of the substrate from the chemical stress generated by the brush and derive a purely mechanical model of the curved substrate.

For an elastic solid under a biaxial strain $\varepsilon$, the elastic free energy is given by, in cylindrical polar coordinates

$$
F=\frac{E^{\prime}}{2} \iiint \varepsilon^{2} r \mathrm{~d} r \mathrm{~d} \phi \mathrm{d} z
$$

where $E^{\prime}$ is the biaxial Young's modulus of the solid, $E^{\prime}=$ $E /(1-v)$ (where $E$ is the linear Young's modulus and $v$ is the Poisson's ratio). Since $\varepsilon$ varies over the thickness of the substrate, integrating over the volume of the substrate gives

$$
F=\frac{E^{\prime}}{2} \int_{z=0}^{w} \int_{\phi=0}^{\theta} \int_{R-(1 / 2) h}^{R+(1 / 2) h} \varepsilon(r)^{2} r \mathrm{~d} r \mathrm{~d} \phi \mathrm{d} z
$$

where $h$ is the thickness of the substrate, $w$ the width in the $z$-direction, $\theta$ the angle subtended by the midline of the substrate, and $R$ the radius of curvature. The strain within the bent substrate is, by definition, equal to the increase in circumference divided by the original circumference. Since the strain at the neutral plane $(r=R$, the midline of the substrate) is zero, the general strain function is

$$
\begin{aligned}
\varepsilon(\mathrm{r}) & =\frac{r \theta-R \theta}{R \theta} \\
& =\frac{r}{R}-1
\end{aligned}
$$

Therefore, the elastic free energy is, substituting into eq 2

$$
F=\frac{E^{\prime} h^{3}}{24 R} w \theta
$$

Since surface stress, $\sigma$, is defined as the change in surface energy per unit area required to create a new surface by elastic deformation, it can be expressed as

$$
\sigma=\frac{\partial F}{\partial S}
$$

where $S$ is the surface area. For a substrate with radius of curvature $R$ and width $w$, the cross-sectional area at a given value of $r$ is given by

$$
A(r)=w r \theta
$$




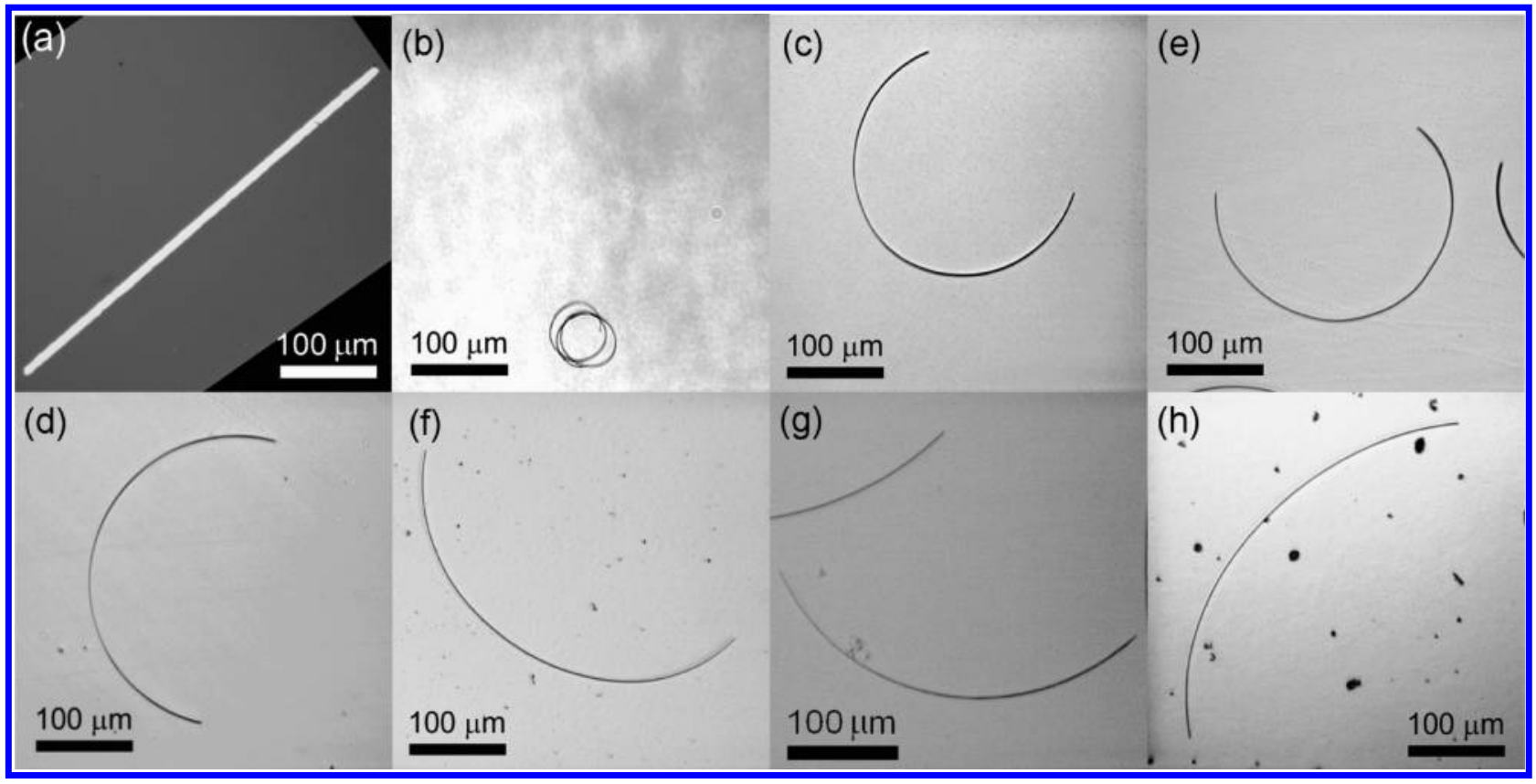

Figure 2. Representative images of (a) a brush-free Au strip in water (the highly reflective bare gold surface is responsible for the apparently inverted contrast) and of Au-brush bilayer objects in pure water. Au thickness: (b) 40, (c) 50, (d) 60, (e) 70, (f) 80, (g) 90, and (h) $100 \mathrm{~nm}$.

Hence, the area of the gold-polymer interface, $S$, at which $r=R+1 / 2 h$ is

$$
S=w \theta\left(R+\frac{h}{2}\right)
$$

The original or unstrained area, $A_{0}$, is given simply by $A_{0}=$ $w R \theta$; hence

$$
\frac{S}{A_{0}}=1+\frac{h}{2 R}
$$

Rearranging and substituting for $R$ in eq 5 gives

$$
F=\frac{E^{\prime} h}{6} \frac{\left(S-A_{0}\right)^{2}}{A_{0}}
$$

Differentiation with respect to $S$ gives the expression for $\sigma$ :

$$
\sigma=\frac{E^{\prime} h}{3}\left(\frac{S}{A_{0}}-1\right)
$$

Substituting eq 9 into eq 11 gives an expression relating $\sigma$ to $R$, which can then be rearranged to give

$$
R=\frac{E^{\prime} h^{2}}{6 \sigma}
$$

Surface charging due to the adsorption of ions is also known to have an effect on the surface stress of gold thin films in salt solutions. ${ }^{33,34}$ In order to eliminate this as a contribution to the surface stress of the bilayer objects, control experiments were carried out. Brush-free gold strips were fabricated by first growing patterned brushes, then performing the gold etching step, then etching away the brushes, and exposing the gold to a solution of the thiol initiator. After $\mathrm{Cr}$ etching, these objects-gold strips coated on one side with a thiol SAM-were washed off the substrate into DI water and 0.1 and $1.0 \mathrm{M} \mathrm{NaCl}$ solutions. These objects showed no curvature in water or salt solution (see Figure 2a), justifying the omission of surface charging effects from the theoretical treatment above.

Experimental Determination of Surface Stress. The freestanding $\mathrm{Au}$-PMETAC objects were imaged in suspension in DI water and in $0.1 \mathrm{M} \mathrm{NaCl}$ solution by optical microscopy. The radii of curvature were measured (see Figures 2 and 3 , respectively) after $24 \mathrm{~h}$ equilibration in suspension. The thickness of the Au layer, $h$, can be controlled during the fabrication process, and the elastic modulus and Poisson ratio are taken to be $54 \mathrm{GPa}$ and 0.45 , respectively. ${ }^{35,36} \mathrm{With}$ these values known, it is possible to fit the experimental curvature data to eq 12 with the surface stress $\sigma$ as the fitting parameter. As shown in Figure 4, eq 12 fits the experimental results well and gives values for the surface stress induced by the charged brush layer: in pure water, $\sigma=0.51 \pm$ $0.03 \mathrm{~N} \mathrm{~m}^{-1}$; in $0.1 \mathrm{M} \mathrm{NaCl}, \sigma=1.90 \pm 0.07 \mathrm{~N} \mathrm{~m}^{-1}$. These values are comparable to the surface stress produced by poly(methacryloxyethylene phosphate) brushes on cantilevers reported by Zhou et al. ${ }^{19}$ The mechanical stress within the brush layer arises from the same free energy balance that causes the vertical stretching of the polymer chains. On a rigid substrate, there is only one way to reduce the interchain repulsion - the chains must stretch vertically. However, on a flexible substrate, there is another potential way to attenuate these repulsive interactions - by substrate deformationand this gives rise to a surface stress. The elements of the surface stress tensor, $\sigma_{i j}$, can be expressed as a function of the osmotic pressure, $\Pi_{\mathrm{os}}$, in the brush, the brush thickness, $H$, and the corresponding element of the strain tensor $\varepsilon_{i j}$, as follows: ${ }^{13,14}$

$$
\sigma_{i j}=\Pi_{\mathrm{os}}\left(\frac{\partial H}{\partial \varepsilon_{i j}}\right)
$$

The osmotic pressure is the driving force for chain extension and may arise from a combination of different factors depending on the scaling regime the brush is in. Since stretching the 


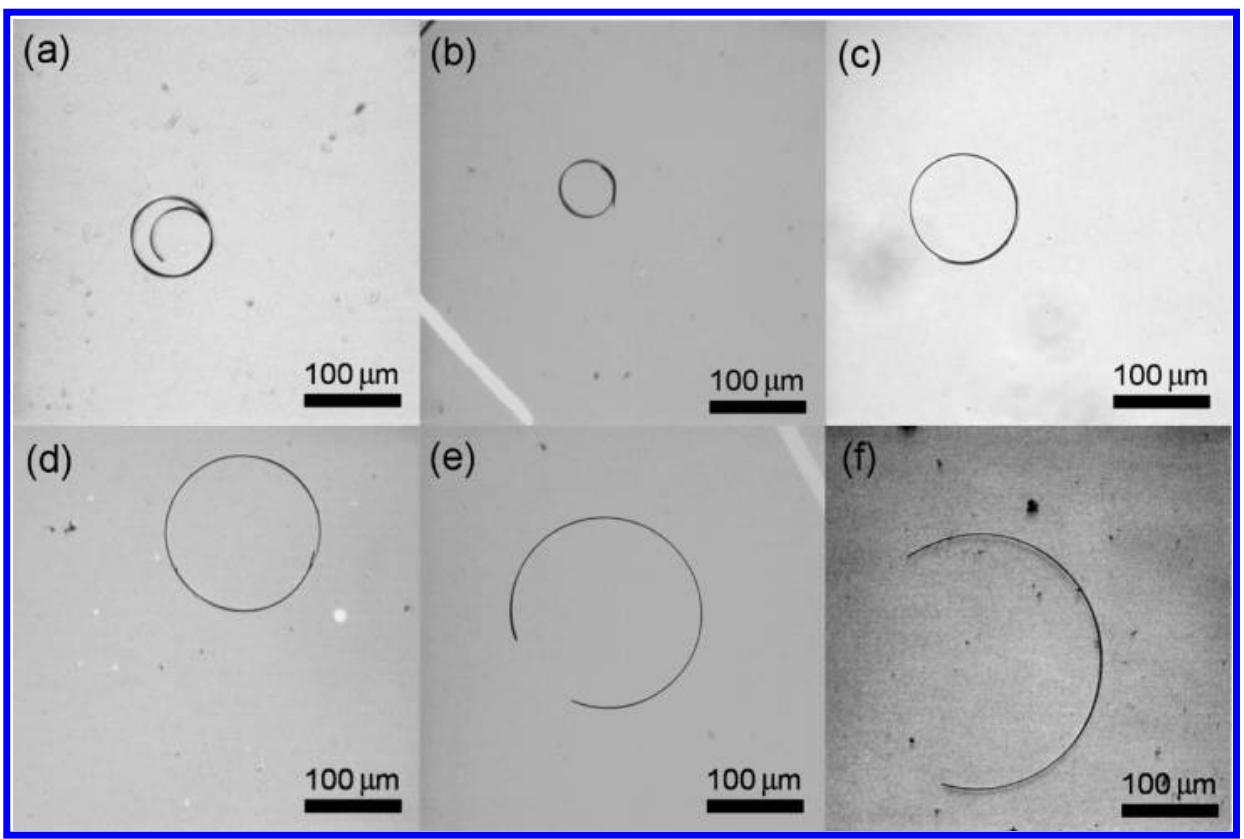

Figure 3. Representative images of Au-brush bilayer objects in $0.1 \mathrm{M} \mathrm{NaCl}$. Au thickness: (a) 60, (b) 70, (c) 80, (d) 90, (e) 100, and (f) $120 \mathrm{~nm}$.

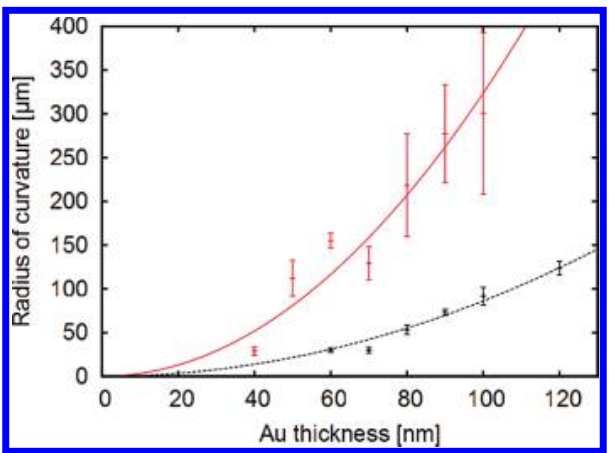

Figure 4. Graph of radius of curvature $R$ against Au thickness for $\mathrm{Au}$-PMETAC bilayers in pure water (red) and in $0.1 \mathrm{M} \mathrm{NaCl}$ solution (black), showing experimental data (points) and model fits based on eq 12 (lines).

substrate causes the distance between the polymer anchoring points to increase, the strain can be expressed as an effective grafting density, $\rho_{\mathrm{eff}}=\rho_{0}\left(1-\varepsilon_{k k}\right)$, where $\rho_{0}$ is the initial grafting density and $\varepsilon_{k k}=\varepsilon_{x x}+\varepsilon_{y y}$ is the first invariant of the strain tensor. Substituting this into eq 13 gives

$$
\sigma_{i j}=-\delta_{i j} \rho_{0} \Pi_{\mathrm{os}}\left(\frac{\partial H}{\partial \rho_{\mathrm{eff}}}\right)
$$

where $\delta_{i j}$ is the Kronecker delta function. The stress is negative because it is compressive and hence causes the substrate to bend away from the brush-this justifies the, until now implicit, assumption that the brush is on the "outside", or positive-strain, side of the curved substrate. Therefore, the brush only generates a surface stress if the brush height depends on the effective grafting density (i.e., the differential of $H$ with respect to $\rho_{\text {eff }}$ is nonzero). Whether this is the case can be determined from scaling theories of polyelectrolyte brushes. If, on a rigid substrate, the brush thickness shows a grafting density dependence, then on a flexible substrate the brush will generate a mechanical stress. For polyelectrolyte brushes in the osmotic regime, ${ }^{37-39}$ which has been observed experimentally for

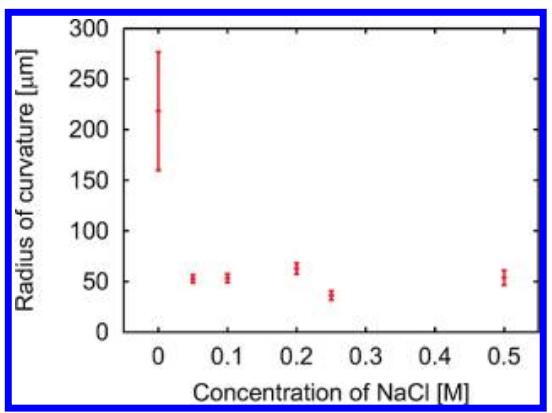

Figure 5. Graph of radius of curvature against salt concentration for $\mathrm{Au}$-PMETAC bilayers in $\mathrm{NaCl}$ solution (Au thickness $80 \mathrm{~nm}$ ).

brushes immersed in a good, salt-free, solvent, ${ }^{40-42}$ the repulsive term in the brush free energy is dominated by the osmotic pressure of the counterions, which are electrostatically confined within the brush. In this regime, simple scaling theories predict a brush thickness independent of grafting density, ${ }^{11,37-39,43}$ so there should be no mechanical stress within salt-free brushes.

Our experimental results show a mechanical stress present even in salt-free water, which contradicts these predictions. However, for high-density polyelectrolyte brushes a nonlinear osmotic regime has been experimentally observed, in which the brush height shows a weak grafting density dependence. ${ }^{44,45}$ Theoretically, this dependence can be accounted for by considering lateral inhomogeneities in the counterion distribution ${ }^{46,47}$ caused by counterion condensation, ${ }^{48}$ explicitly including the polymer excluded volume, ${ }^{44,47}$ and by using a nonlinear elastic model of chain stretching. ${ }^{47}$ Since the grafting density dependence is weak, less stress should result for a brush in pure water compared to a brush in the salted regime. The effect of salt concentration can be seen from Figure 5. The transition from the nonlinear osmotic regime (low stress) to the salted brush regime (high stress) occurs at low salt concentration-between 0 and $0.05 \mathrm{M} \mathrm{NaCl}$. According to scaling theory, the critical salt concentration that defines the boundary between the osmotic and salted regimes scales with the inverse of grafting density. ${ }^{38,39}$ 


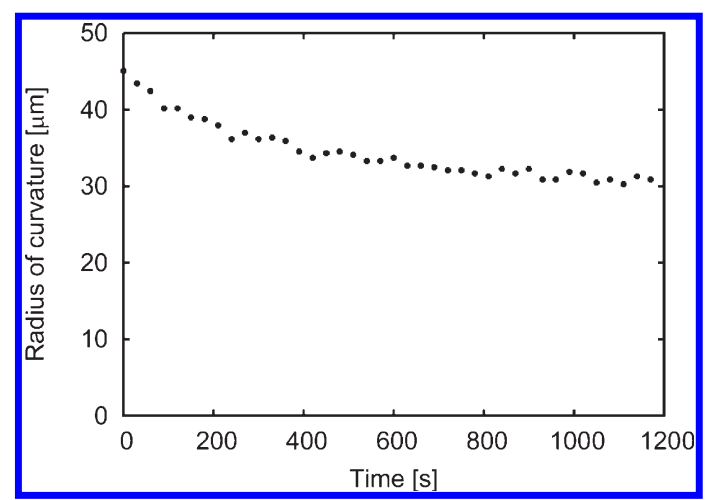

Figure 6. Graph of radius of curvature over time for Au-PMETAC bilayers in pure water (Au thickness $40 \mathrm{~nm}$ ).

The response time of the brushes can be determined by taking successive optical microscopy images of a single bilayer object over time (see Figure 6). This method is imperfect due to the difficulty of defining a time zero-after the objects are washed off the $\mathrm{Si}$ wafer, which takes 1-2 s, several seconds may elapse before the suspension becomes stable enough to image. However, this technique gives an idea of the time scale of the swelling transition, which is $\sim 5 \mathrm{~min}$. This is in agreement with previous work, which has shown a long response time, of the order of minutes, for brush-modified cantilevers. ${ }^{49}$

\section{Conclusion}

Free-standing $\mathrm{Au}$-PMETAC thin-film bilayers provide a simple, easily fabricated platform for examining and quantifying the mechanical stresses present in stimulus-responsive polyelectrolyte brushes. For the dense, strongly charged brushes in this study, deviation is observed from the osmotic regime predicted by simple scaling theories - instead, these brushes must be modeled by a more complex nonlinear treatment. The mechanical behavior of the $\mathrm{Au}$-PMETAC bilayer system is to be understood in terms of surface stresses which arise from the interplay between the brush free energy and the elastic deformation energy of the substrate. This is in contrast to the approach taken by Sushko ${ }^{15}$ in calculating the deflection of cantilevers modified with polyelectrolyte brushes, which used DFT calculations to determine the parameters of the brush but treated the surface stress as arising from purely electrostatic considerations. Understanding the mechanical behavior of polyelectrolyte brushes is a vital step toward realizing the potential of stimulus-responsive polymer brushes as a platform for developing components for soft nanotechnology. ${ }^{50}$ In particular, this work has implications for the development of polyelectrolyte brushes as nanoactuators ${ }^{18}$ and for mechanosensitive surfaces ${ }^{51}-$ areas in which an understanding of the mechanical properties of these systems, and of how chemical processes give rise to and modify mechanical stress, is vital.

Acknowledgment. This research has been supported by the Engineering and Physical Sciences Research Council (EPSRC) and a Friedrich Wilhelm Bessel Research Award of the Humboldt Foundation to W.T.S.H.

\section{References and Notes}

(1) Ballauff, M.; Borisov, O. Curr. Opin. Colloid Interface Sci. 2006, 11, 316-323.

(2) Rühe, J. et al. Polyelectrolyte brushes. In Polvelectrolvtes with Defined Molecular Architecture I: Springer: Berlin, 2004; Vol. 165, pp 79-150.

(3) Wilbur, J. L.; Kumar, A.; Kim, E.; Whitesides, G. M. Adv. Mater. 1994, 6, 600-604
(4) Xia, Y.; Whitesides, G. M. Langmuir 1997, 13, 2059-2067.

(5) Azzaroni, O.; Moya, S.; Farhan, T.; Brown, A. A.; Huck, W. T. S. Macromolecules 2005, 38, 10192-10199.

(6) Zhou, F.; Huck, W. T. S. Phvs. Chem. Chem. Phvs. 2006, 8, 3815-3823.

(7) Ayres, N.; Boyes, S. G.; Brittain, W. J. Langmuir 2007, 23, 182-189.

(8) Ayres, N.; Cyrus, C. D.; Brittain, W. J. Langmuir 2007, 23, 3744-3749.

(9) Yu, K.; Wang, H.; Xue, L.; Han, Y. Langmuir 2007, 23, 1443-1452.

(10) Milner, S. T. Science 1991, 251, 905-914.

(11) Naji, A.; Seidel, C.; Netz, R. Theoretical Approaches to Neutral and Charged Polymer Brushes. In Surface-Initiated Polvmerization II: Springer: Berlin, 2006; pp 149-183.

(12) Lyatskaya, Y.; Balazs, A. C. Macromolecules 1996, 29, 5469-5474.

(13) Begley, M. R.; Utz, M.; Komaragiri, U. J.Mech. Phvs. Solids 2005, $53,2119-2140$

(14) Utz, M.; Begley, M. R. J. Mech. Phvs. Solids 2008, 56, 801-814.

(15) Sushko, M. L. Faradav Discuss. 2009, 143, 63-80.

(16) Ryan, A. J.; Crook, C. J.; Howse, J. R.; Topham, P.; Geoghegan, M.; Martin, S. J.; Parnell, A. J.; Ruiz-Pérez, L.; Jones, R. A. L. J. Macromol. Sci., Part B: Phvs. 2005, 44, 1103-1121.

(17) Comrie, J. E.; Huck, W. T. S. Macromol. Rapid Commun. 2008, 29, 539-546.

(18) Huck, W. T. S. Mater. Todav 2008, 11, 24-32.

(19) Zhou, F.; Shu, W. M.; Welland, M. E.; Huck, W. T. S. J. Am. Chem. Soc. 2006, 128, 5326-5327.

(20) Zhou, F.; Biesheuvel, P. M.; Chol, E. Y.; Shu, W.; Poetes, R.; Steiner, U.; Huck, W. T. S. Nano Lett. 2008, 8, 725-730.

(21) Bumbu, G. G.; Wolkenhauer, M.; Kircher, G.; Gutmann, J. S.; Berger, R. Langmuir 2007, 23, 2203-2207.

(22) Abu-Lail, N. I.; Kaholek, M.; LaMattina, B.; Clark, R. L.; Zauscher, S. Sens. Actuators, B 2006, 114, 371-378.

(23) Ji, H.-F.; Armon, B. D. Anal. Chem. 2010, 82, 1634-1642.

(24) Pei, Q.; Inganäs, O. J. Phvs. Chem. 1992, 96, 10507-10514.

(25) Pei, Q.; Inganäs, O. Solid State Ionics 1993, 60, 161-166.

(26) Christophersen, M.; Shapiro, B.; Smela, E. Sens. Actuators, B 2006, $115,596-609$.

(27) Jones, D. M.; Brown, A. A.; Huck, W. T. S. Langmuir 2002, 18, 1265-1269.

(28) Edmondson, S.; Huck, W. T. S. Adv. Mater. 2004, 16, 1327-1331.

(29) Comrie, J. E.; Huck, W. T. S. Langmuir 2007, 23, 1569-1576.

(30) Wang, M.; Comrie, J. E.; Bai, Y.; He, X.; Guo, S.; Huck, W. T. S. Adv. Funct. Mater. 2009, 19, 2236-2243.

(31) Li, Y.; Armes, S. P.; Jin, X.; Zhu, S. Macromolecules 2003, 36, $8268-8275$.

(32) Ye, J.; Narain, R. J. Phvs. Chem. B 2009, 113, 676-681

(33) Tabard-Cossa, V.; Godin, M.; Beaulieu, L.; Grütter, P. Sens. Actuators, B 2005, 107, 233-241.

(34) Smetanin, M.; Viswanath, R. N.; Kramer, D.; Beckmann, D.; Koch, T.; Kibler, L. A.; Kolb, D. M.; Weissmüller, J. Langmuir 2008, 24, 8561-8567.

(35) Espinosa, H. D.; Prorok, B. C. J. Mater. Sci. 2003, 38, 4125-4128.

(36) Faurie, D.; Renault, P.-O.; Le Bourhis, E.; Goudeau, P. Acta Mater. 2006, 54, 4503-4513.

(37) Borisov, O. V.; Birshtein, T. M.; Zhulina, E. B. J. Phvs. II 1991, 1, $521-526$.

(38) Israëls, R.; Leermakers, F. A. M.; Fleer, G. J.; Zhulina, E. B. Macromolecules 1994, 27, 3249-3261.

(39) Borisov, O. V.; Zhulina, E. B.; Birshtein, T. M. Macromolecules 1994, 27, 4795-4803.

(40) Ahrens, H.; Förster, S.; Helm, C. A. Phvs. Rev. Lett. 1998, 81, 4172-4175.

(41) Tran, Y.; Auroy, P.; Lee, L.-T. Macromolecules 1999, 32, 8952-8964.

(42) Biesheuvel, P. M. J. Colloid Interface Sci. 2004, 275, 97-106.

(43) O'Shaughnessy, B.; Yang, Q. Europhvs. Lett. 2006, 75, 427-433.

(44) Ahrens, H.; Forster, S.; Helm, C. A.; Kumar, N. A.; Naji, A.; Netz, R. R.; Seidel, C. J. Phvs. Chem. B 2004, 108, 16870-16876.

(45) Romet-Lemonne, G.; Daillant, J.; Guénon, P.; Yang, J.; Mays, J. W. Phvs. Rev. Lett. 2004, 93, 148301

(46) Seidel, C. Macromolecules 2003, 36, 2536-2543.

(47) Naji, A.; Netz, R.; Seidel, C. Eur. Phvs. J. E 2003, 12, 223-237.

(48) Hehmeyer, O. J.; Arya, G.; Panagiotopoulos, A. Z.; Szleifer, I. J. Chem. Phvs. 2007, 126, 244902.

(49) Valiaev, A.; Abu-Lail, N. I.; Lim, D. W.; Chilkoti, A.; Zauscher, S. Langmuir 2007, 23, 339-344.

(50) Cohen Stuart, M. A.; Huck, W. T. S.; Genzer, J.; Müller, M.; Ober, C.; Stamm, M.; Sukhorukov, G. B.; Szleifer, I.; Tsukruk, V. V.; Urban, M.; Winnik, F.; Zauscher, S.; Luzinov, I.; Minko, S. Nature Mater. 2010, 9, 101-113.

(51) Bünsow, J.; Kelby, T. S.; Huck, W. T. S. Acc. Chem. Res. 2009. 